\title{
Aplication of new MBBR Packing in Wastewater Treatment
}

\author{
Jianhua Yang ${ }^{1, a}$, Li Ye $\mathrm{C}^{2, \mathrm{~b}}$ and Yi Fan ${ }^{3, \mathrm{c}}$ \\ ${ }^{1}$ Chongqing Institute of Mechanical \& Electrical Engineering, Long Xi Song Pai Road $98 \mathrm{HaO}, \mathrm{Yu}$ \\ Bei , Chongqing , 401147, China \\ ${ }^{2}$ Chongqing Institute of Mechanical \& Electrical Engineering, Long Xi Song Pai Road $98 \mathrm{HaO}, \mathrm{Yu}$ \\ Bei , Chongqing , 401147, China \\ ${ }^{3}$ Chongqing Institute of Mechanical \& Electrical Engineering, Long Xi Song Pai Road $98 \mathrm{HaO}$, Yu \\ Bei , Chongqing , 401147, China \\ a,b,c624525078@qq.com
}

Key words: wastewater treatment; MBBR packing; environmental protection

Abstract. the problem of environmental protection is a hot topic in recent years, the sewage treatment is an important aspect of environmental protection.In view of China's current sewage treatment problems, a new MBBR filler for MBBR process can improve the sewage treatment effect.

\section{Introduction}

Environmental protection is a hot topic at present. It is urgent to protect the water quality. Although the increasing degree of attention to environmental protection, and sewage treatment volume also increased year by year, but the rapid expansion of China current sewage treatment capacity is not the size of the water.The sewage treatment rate is low.

\section{Sewage treatment status of domestic}

\section{sewage treatment technology}

Generally ,sewage treatment use the traditional wastewater treatment technology, such as flocculation, sand filtration system, adding ferric chloride reagent to design $\mathrm{A} 2 / \mathrm{O}$ system final sedimentation tank with water wells, enhanced biological phosphorus removal, reducing the concentration of phosphorus in the final effluent. A water pumping station after precipitation to the sand filter, the air filter backwash water, filtered water to clean water tank, pressurized into the reuse water network. In the traditional sewage treatment system, the sedimentation tank is used to treat the wastewater. But,it can not form a good condition of particle agglomeration and can not produce aggregate flocs. This can lead to lowly solid-liquid separation efficiency.

\section{common packing}

Filler is a key part in the process of sewage treatment. The material, having different characteristics, are used different water.In the process of packing selection, according to the different water quality and treatment process, select the appropriate filler.

In recent years, domestic research on suspended biological filler are gradually mature. The gradully filler used in China include cellular filler, soft filler, semi soft packing machine composite filler fixed type filler. But these are many problems when the filler is using, such as agglomeration, gas distribution and water distribution is not uniform. This will effect of the biological treatment effect. Moreover, all of the fillers need to be installed on the auxiliary support, which will cause inconvenience to the installation and replacement of the filler.And the project investment and operation management cost will be increased.

\section{Characteristic of mbbr filler}

In recent years, many domestic scholars have devoted themselves to the research of MBBR technology.But the research on MBBR filler has just started. MBBR filler is a microbial carrier which 


\section{ATLANTIS
PRESS}

are used in the MBBR process. It is mainly to provide a suitable environment for microbial growth. MBBR filler is a new bioactive carrier.It uses scientific formula, according to the different nature of the sewage in polymer materials, integration of a variety of trace elements is conducive to rapid attachment of microbial growth, through special process modification, structure, has the advantages of large specific surface area, hydrophilic, high biological activity, biofilm fast, good effect, long service life etc.. Filler performance is evaluation of the most important indicators of the amount of biological filler filler.

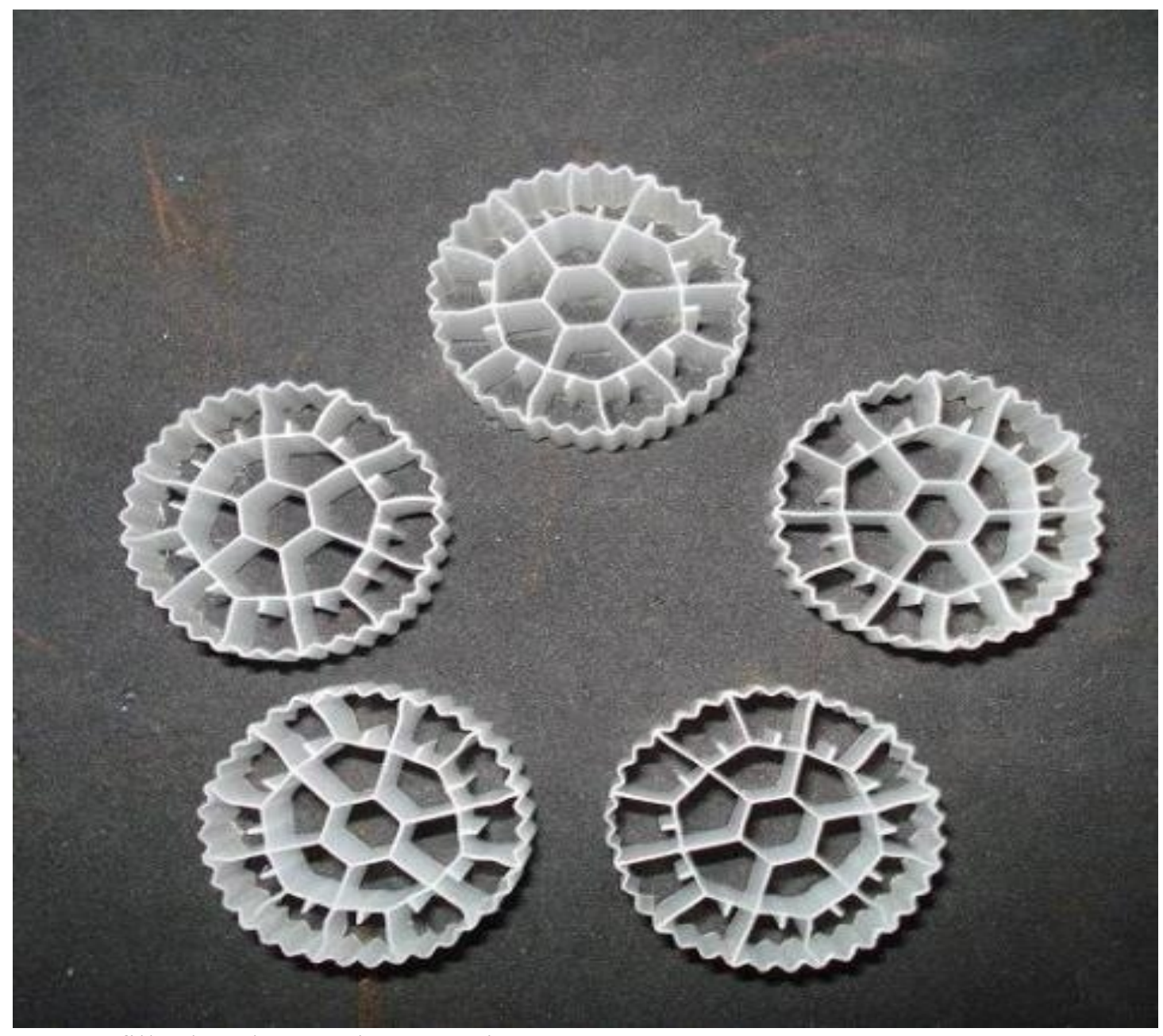

MBBR filler has three main properties:

\section{Surface properties of filler}

(1).surface structure: it is generally considered have the surface roughness, film fast.

(2).the surface potential: General microorganisms with negative charge, filler surface is positive for microbial growth.

(3). hydrophilic: microorganisms as hydrophilic particles, fillers hydrophilic good for microbial growth.

\section{hydraulic performance}

(1). porosity: the volume of the filler, high porosity.

(2). shape and size: the flow of water, air flow

(3) fluidization performance: related to packing density: The density of the packing should be 0.97-1.03, which can be realized by small aeration or stirring. 


\section{MBBR process for treating wastewater}

\section{process principle}

Water tank after pretreatment by the coarse grid and the fine grid interception, then entering the water regulating tank, the regulating tank to the integrated sewage treatment plant sewage biochemical treatment by the lift pump, through the anoxic and aerobic MBBR treatment and two sedimentation tank solid-liquid separation. Finally, effluent after AFF asymmetric fiber filtration system and ultraviolet disinfection treatment, discharge standards.

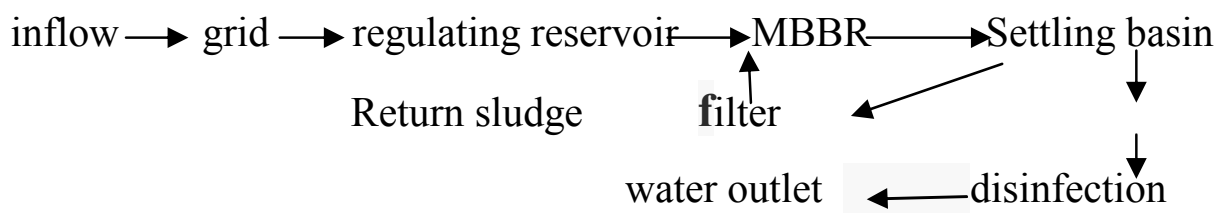

When the aeration, the biofilm packing density and water due to close packing growth, stirring rely on aeration, in a fluidized state, which not only makes the biofilm wastewater and filler on the extensive and frequent contact, and the filler in the process of cutting and dispersed bubble flow, the gas distribution tends to be uniform, the use of oxygen the rate has also been improved, fully mixed contact and collision of solid, liquid and gas phase of the resulting increases the mass transfer area, increasing the mass transfer rate, strengthen mass transfer process. Therefore, in the case of a certain pollutant removal rate, the residence time of the sewage in the MBBR is shorter, even if there is an impact load, it can recover the treatment effect quickly. In addition, by suspended packing airflow, water erosion, aging of the film can automatically fall off, ensure the membrane activity, and promote the The new supersedes the old. in the reactor, with the flow of a large number of filamentous bacteria growth may also be on the packing, both high degradation of organic matter by filamentous bacteria, improve the effluent, and no sludge bulking.

As the proportion of filler and water is similar, only a very small amount of gas can be evenly suspended in water. When the utility model is used, the utility model does not need to be filled with a bracket, only a grid network interception is arranged at the water outlet of the aeration tank, and the water flows back to the front end of the pool by the aeration flow, which can save investment and is convenient to be matched and updated. In addition, the operator does not need to worry about the sludge recycle ratio, the amount of excess sludge and sludge bulking, such as the management of activated sludge system, therefore, the operation is simple, maintenance management, the workload is less.

\section{Application of MBBR filler}

MBBR technology is the core of MBBR suspended packing, aerobic and anaerobic metabolic activity, good nitrogen and phosphorus removal. Biofilm MBBR mainly fixed on the packing, sludge retention time and hydraulic retention time independent, microbial denitrifying bacteria, nitrosobacteria growth generation time longer, much smaller than the growth rate can grow on the packing, so as to enhance the ability of nitrogen removal. The denitrification process is divided into two stages: nitrification and denitrification. MBBR can realize the independent growth of nitrifying bacteria and denitrifying bacteria in space, so as to optimize the growth conditions of two kinds of bacteria.

\section{Conclusions}

Overall, China's current research on suspended filler has just started, MBBR suspended filler in China's sewage treatment engineering application has a wide range of development space. From the point of view of economic, practical and efficient, high performance new packing materials, should have the characteristics of low price, long service life, easy biofilm; in terms of structure, design the specific surface area should be as large as possible, and can make some functional areas, to adapt to 
the different requirements of anaerobic and aerobic the growth of microorganisms, and easy stripping of the gu. At the same time, as far as possible to reduce the cost of suspended filler, to maximize its advantages, so that MBBR suspended filler can be widely used in sewage treatment.

\section{References}

[1] M.X.Loukidou, A.LZouboulis. Comparison of Biological Treatment Process using Attached-growth Biomass for Sanitary Landfill Leachate Treatment[J]. Environmrntal pollution,2001,11(1):273-281

[2] Horn H, Morgenroth E. Transport of oxygen, sodium chloride, and sodium nitrate in biofilms[J]. Chemical Engineering Science ,2006,61(5):1347-1356

[3] Junliang Liu and Qing Wang. Water treatment packing and filter material[M]. Chemical industry press, 2010

[4] C.S.RA et al. Biological Nutrient Removal with an Internal Origanic Carbon Source in Piggery Wastewater Treatment [J]. Wat. RES.,2000,34(3)965-973 